\title{
Growth, characterization, and antibacterial studies of L-Lysine single crystals added with potassium bromide
}

\author{
P. Yasotha ${ }^{1}$, R. Thiagarajan ${ }^{2}$, P. Sagunthala ${ }^{3}$ \\ ${ }^{1,3}$ Department of Physics, Sri Vasavi College, Erode - 638 316. Tamil Nadu, India. \\ ${ }^{2}$ Department of Physics, Chikkaiah Naiker College, Erode, Tamil Nadu, India
}

\begin{abstract}
Single crystals of L-lysine added Potassium Bromide were grown by slow evaporation technique at room temperature. The crystalline nature of the grown crystal wasconfirmed using powder X-ray diffraction technique. Single crystal X-ray diffraction patterns were recorded for the structural conformation and it was found to be cubic. The UV-VIS-NIR Spectrum of the grown crystals shows less optical absorption and good transmittance in the entire visible region enabling its use in optical applications. Vickers micro hardness test was carried out to analyze the mechanical property of the grown L- lysine potassium Bromide single crystal. Thermo gravimetric analysis proved that the crystal is stable up to $600^{\circ} \mathrm{C}$. The frequency and temperature dependence of dielectric constant $\left(\varepsilon_{r}\right)$, dielectric loss (tan $\delta$ ) were also measured. The grown crystal was evaluated for its biological efficacy and found to exhibit anti bacterial activities against some select bacterial strains.
\end{abstract}

Keywords: Anti bacterial, Dielectric studies, Micro Hardness, Powder X-Ray Diffraction, Slow Evaporation,

\section{Introduction}

Amino acids are the building blocks of proteins that are very important for most of the processes in livingorganisms [1] and their complexes belong to a class of organicmaterials find immense nonlinear optical (NLO) applications [2].Recently, complexes of amino acids have been explored. The tetrahedral array of four different groups about a- carbon atom confersoptical activity of amino acid. In solid state, amino acid contains a deprotonated carboxyl acid group $\left(\mathrm{COO}^{-}\right)$andprotonated amino group $\left(\mathrm{NH}^{3+}\right)$. This zwitter ionic nature helps to increase thecrystal hardness, thus making them idealcandidates for NLO devices [3]Complexes of amino acids with inorganic acids and salts are promising materialsfor optical second harmonic generation (SHG), as they tend to combine the advantages of the organic amino acidwith that of the inorganic acid [4]. In recent years, efforts have been made to synthesize amino acid mixedinorganic complex crystals, in order to improve the chemical stability, laser damage threshold, linear and nonlinear optical properties. The present investigation deals with the growth of L-lysine added Potassium Bromide singlecrystal by slow solvent evaporation technique. The grown crystal has been subjected to powder XRD, single crystal XRD, UV-VIS, micro hardness and dielectricstudies. The grown crystals were also screened for anti bacterial studies toidentify the application area of the material.

\section{Experimental Method}

High purity (AR grade 99\% purity) Merck grade salts were used for the preparation. Saturated solutions ofPotassium Bromideand L-lysine were prepared separately by adding the salts gradually and stirred using magnetic stirrer (Remi-1 MLH) and then filtered twice using Whatman no:1 filter paper. The above prepared saturated solutions of $\mathrm{KBr}$ and $\mathrm{L}$ - lysine were mixed in the ratio (3:1) and taken in a well cleaned beaker. The solution was stirred well for two hours to get the homogeneity. The beakerwas closed with perforated aluminum foil and kept in a dust free and vibration free environment and observed periodically. After a time span of 95 days the L- lysine added Potassium Bromidesingle crystal (LYPB)was harvested successfully. The photographs of the growncrystals were shown in the figure 1.

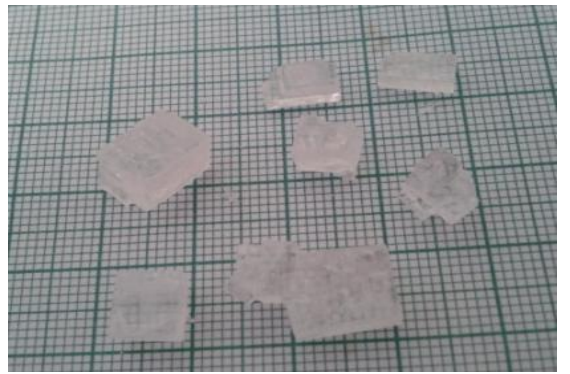

Figure 1. Photograph of the grown crystal 
Growth, characterization, and antibacterial studies of L-Lysine single crystals added with ..

\subsection{Powder X-ray diffraction}

\section{Results And Discussions}

The grown crystalsof LYPB were subjected to powder X-ray diffraction analysis. The powder X-ray spectra were recorded using SEIFERT X-ray diffractometer with $\mathrm{CuK} \alpha$ Radiation $(\lambda=1.5406)$. The powdered samples were scanned over the range of 10 to 80 degrees. The peaks in the diffractogram were indexed using powder X-ray software. The XRD pattern of the grown crystals of LYPB is shown in the fig.2. The presence of number of good intensity and sharp peaks prove the crystalline nature of the grown crystals $[5,6]$.

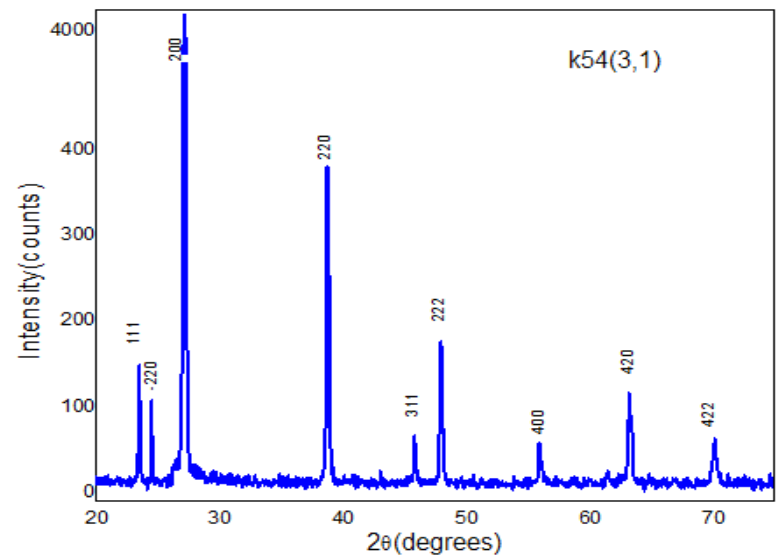

Figure: 2 PXRD pattern of grown crystals

\subsection{Single crystal $\mathrm{X}$-ray diffraction analysis}

The single X-ray diffraction studies have been carried out to confirm the crystalline nature and to determine the lattice parameters of the grown sample. It has been carried out using Bruker AXS diffractometer using MoK $\alpha$ radiation $(\lambda=0.71073 \AA)$. From the SXRD data it is observed that thegrown LYPB crystals belongto cubic crystal system. The calculated lattice parameter values are presented in table 1 . The results of the present work are compared with that of pure $\mathrm{KBr}[7,8]$. The Lysine added with potassium bromide did not alter the structure but there is a mild change in the lattice parameter values there by increasing the volume of the unit cellwhich proves the incorporation of Lysine into the lattice sites of potassium bromide.

TABLE 1. Cell parameters of the grown crystals

\begin{tabular}{|c|c|c|c|c|c|c|}
\hline Crystal & $\mathbf{a ~ ( \square )}$ & $\mathbf{b}(\square)$ & $\begin{array}{c}\text { c } \\
\mathbf{\square}\end{array}$ & $\square=\square=\square$ & Volume ( $\square$ ) & Crystal system \\
\hline $\mathrm{KBr}$ & 6.59 & 6.59 & 6.59 & $90^{\circ}$ & 287.05 & cubic \\
\hline $\mathrm{LYPB}$ & 6.79 & 6.79 & 6.79 & $90^{\circ}$ & 314 & cubic \\
\hline
\end{tabular}

\subsection{UV-vis-NIR spectral analysis}

The optical absorption spectrum of LYPB crystal was recorded in the wavelength range of 200-1000 $\mathrm{nm}$ using Perkin Elmer Lamda 935 UV-vis-NIR spectrometer. The obtained absorption spectrum is shown in Fig 3. in whichthe lower cut off region is obtained at $246 \mathrm{~nm}$.

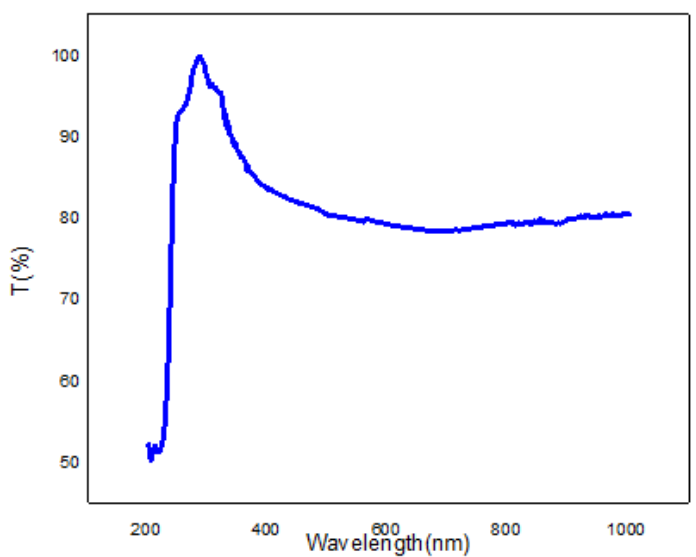

Figure 3. UV Transmission graph of grown crystal 
The UV spectra shows the presence of a wide transparency window lying between 246 and $800 \mathrm{~nm}$ with $\lambda_{\min }=246 \mathrm{~nm}$.. Using the relation $\mathrm{E}_{\mathrm{g}}=1240 / \lambda_{\min }$, the band gap energy wasfound to be $5.04 \mathrm{eV}$. The optical absorption spectrum shows that absorption was very less in theentire visible region and part of IR region [9].Hence, from the analysis of absorption spectrum, it is evident that the grown crystal is transparent in the entire visible region without any absorption peak, which is the key requirement for any nonlinear optical crystal having applications in second harmonic generation, parametric oscillations, etc [10,5].The ideal crystalline materials for band-pass filters should be narrow band transmissive in specific wavelength and it can be used as sensors for aircraft, spaceship and to estimate missile applications.

\subsection{Mechanical properties: micro hardness studies}

One of the methods to determine the mechanical behavior of the grown crystals is micro hardness test. The polished surface of the grownLYPB crystals were indented at different sites for the loadfor 10 seconds[11]. Indentations were made using pyramidal diamond indentor by varying the loads from 25 to $100 \mathrm{~g}$. The successive indentations were made at different sites of the plane. The mean diagonal length was used for calculating the Vickers's hardness number. The hardness test could not be carried out above $100 \mathrm{~g}$ because crack initiation and materials chipping become significant beyond this load.
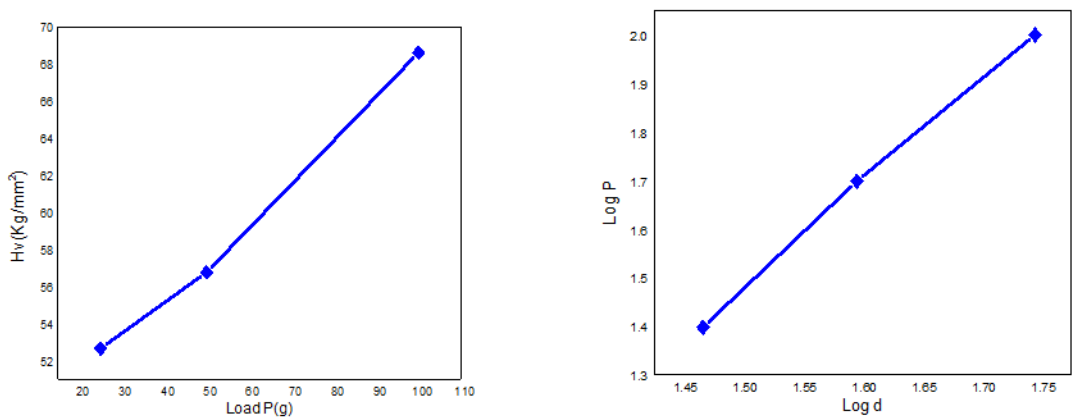

Figure 4a. Variation of $\mathrm{H}_{\mathrm{v}}$ Vs load Figure4b. Variation of $\log \mathrm{P}$ Vs Log d

The Vickers hardness number $\left(\mathrm{H}_{\mathrm{v}}\right)$ was calculated using the relation

$\mathrm{H}_{\mathrm{v}}=1.8344 \mathrm{P} / \mathrm{d}^{2} \mathrm{~kg} / \mathrm{mm}^{2}$

where,

$\mathrm{P}$ - the applied load in grams

$\mathrm{d}$ - the diagonal length of the indentation impression in $\mathrm{mm}[12]$

The micro hardness and the diagonal length were calculated from the micro computer attached to the instrument. Plot of Hvagainst load P for the grown LYPB crystal is shown in figure4a. The non linear variation of $\mathrm{Hv}$ with load implies the presence of imperfection and voids. The imperfections are mainlydue toimpurity, dislocation or grain boundary diffusion.The Meyer's index number n[13] gives the value of work hardening index. Materials are normally characterized by Meyer's index or work hardening index. The lower the value of the work hardening index better will be the hardness of the material[14]. From careful observations on various materials, Onitsch pointed out that the value of nlies between 1 and 1.6 for hard materials and it is morethan 1.6 for soft materials[15]. The log-log plot between $\mathrm{d}$ and $\mathrm{P}$ is shown in the fig $4 \mathrm{~b}$ which is almost a straight line graph. The slope of the line gives the work hardening index $\mathrm{n}$. The $\mathrm{n}$ value obtained for the sampleis 2.08. Hence the value of work hardening index suggest that material is soft.

\subsection{Thermal analysis.}

The grown LYPB crystal was subjectedto heating at the rate of $10^{\circ} \mathrm{Cper}$ min in the nitrogen atmospherefrom $20^{\circ} \mathrm{C}$ to $700^{\circ} \mathrm{C}$. The thermogram is shown in Fig 5 .

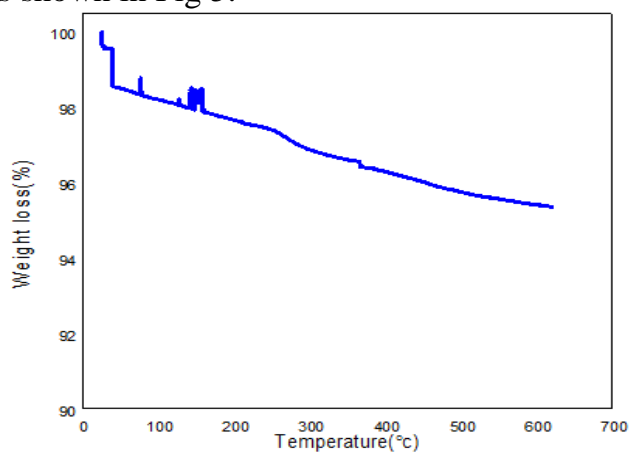

Figure 5. TGA spectra of grown crystal 
The thermogram shows that the weight loss startsafter $100^{\circ}$ Cand below this temperature weight loss is very minimum. Hence the crystal is completely devoid of any inclusion of water, which was used as the solvent for crystallization[16]. It is observed that only $4 \%$ of the sample got disintegrated up to $400^{\circ} \mathrm{C}$. There is a gradual weight loss of $1 \%$ of the sample upto $600^{\circ} \mathrm{C}$ and about 95 percentage of the LYPB crystal remains stable. From these results, it is concluded that the crystalis thermally stable up to $600^{\circ} \mathrm{C}$. The stability of this crystal is a desirable property for its possible NLO applications.

\subsection{Dielectric studies}

The dielectric characteristics of the material are important to study the lattice dynamics in the crystal[17].The grown crystalLYPB was subjected to dielectric studiesat different temperatures like $30^{\circ} \mathrm{C}, 40^{\circ} \mathrm{C}, 60^{\circ} \mathrm{C}, 80^{\circ} \mathrm{C}$ and $100^{\circ} \mathrm{C}$. The variation of dielectric constant and dielectric loss as a function of frequency with varyingtemperature is shown in the figure 6 and figure 7.

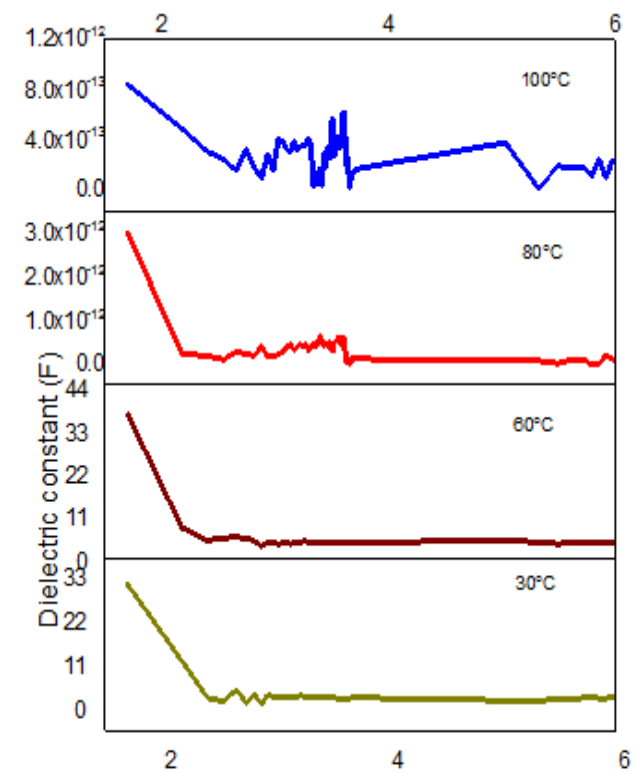

Fig 6. Dielectric constantVs frequency

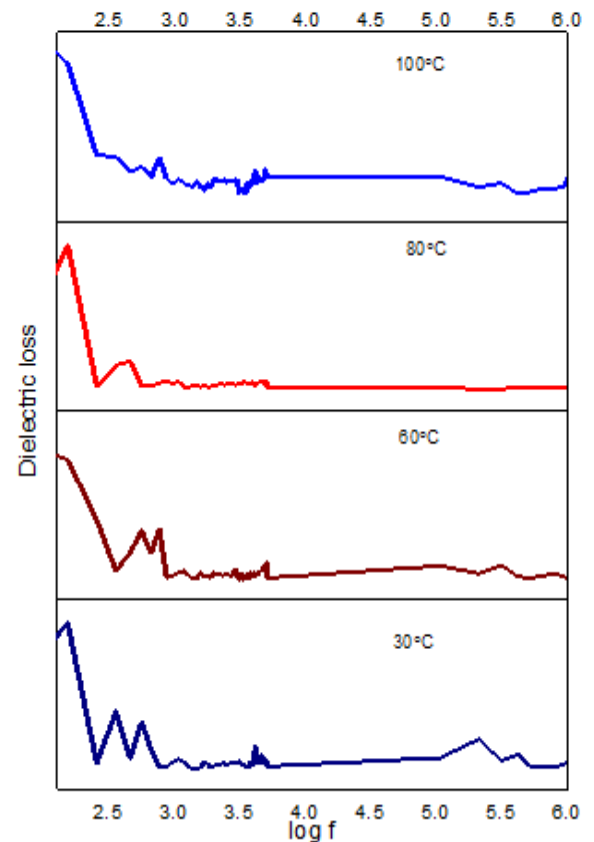

Fig 7. Dielectric loss Vs frequency

The dielectric constant is higher at low frequencies and then decreases with the increasing frequencies and saturates and dielectric loss decreases with increasing frequency. The large value of dielectric constant at low frequency is due to the presence of space charge polarization.

At lower frequencies all the polarizations are active whereas in the case of high frequencyonly electronic and ionic polarizations are active. When the electric charge carriers cannot follow the alteration of the a.c. electric field applied beyond a certain critical frequency the dielectric constant decreases with increase in frequency and remains constant [18 -20]. The maximum value of dielectric constant increases with the increasing temperature. At high temperature like $100^{\circ} \mathrm{C}$ there is no smooth decrease in the value of dielectric constant and dielectric loss with increase in frequency. The exchange of the charge carriers in the lattice sites which is responsible for electrical conduction is thermally activated by increasing temperature. As a result, dielectric polarization increases causing an increase in dielectric constant and dielectric loss. It is evident from figure that the crystal has a very low dielectric loss in the high frequency region, which indicates the lesser number of defects in the crystals.

The dielectric loss is strongly dependent on the frequency of the applied field[21]. These results are correlated with piezoelectric results. The very low value of dielectric constant at higher frequencies is important for the fabrication of materials towards ferroelectric, photonic and electro-optic devices and it possesses enhanced optical quality with lesser defects, this parameter is of vital importance for nonlinear optical applications. 
Growth, characterization, and antibacterial studies of L-Lysine single crystals added with ..

\subsection{Antibacterial study (Agar diffusion method)}

Antibiotic resistance has become a global concern. The clinical efficacy of many existing antibiotics is being threatened by emergence of multi drug resistant pathogens. So it is necessary to discover new antimicrobial materials with novel mechanism of action towards new and re-emerging infectious diseases. Antibacterial activities of the grown crystals were analyzed against bacterial strains Lactobacillus, Bacillus subtilis, Staphylococcus aureus, Salmonella typhi and Escherichia coli.

\section{Agar diffusion method}

Antibacterial assay was used to determine the growth inhibition of bacteria. Bacteria were maintained at $4^{0} \mathrm{C}$ on broth media before use. Agar diffusion is a widely accepted in vitro investigation method for preliminary screening of test microorganisms[22].The required amount of petri plates are prepared and autoclaved at $121^{\circ} \mathrm{C}$ for 15 minutes. And they were allowed to cool under laminar air flow. About $20 \mathrm{ml}$ of media was aseptically transfered into each sterile petridishes and allowed to solidify. $0.2 \mathrm{ml}$ inoculum suspension was spread uniformly over the agar medium using sterile L shaped glass rod to get uniform distribution of bacteria.

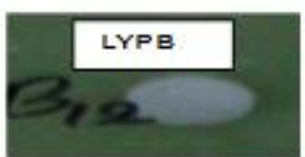

L.bacillus

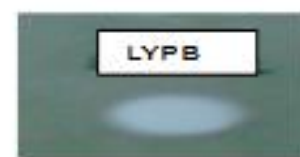

S.aureus

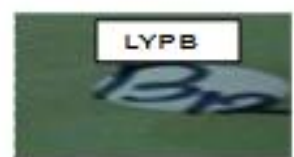

S.typhi

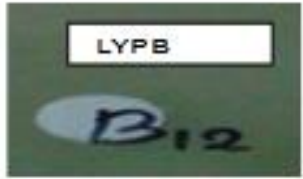

E.coli

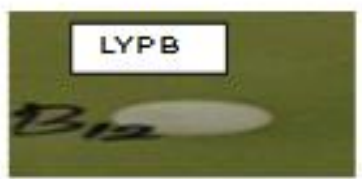

B.subtilus

Figure 8. Zone of inhibition ( $\mathrm{mm}$ ) against select bacteria

The readily prepared cavity were loaded with sample and the plates were incubated at $5^{\circ} \mathrm{C}$ for 1 hour to permit good diffusion and then transferred to an incubator at $37^{\circ} \mathrm{C}$ for 24 hours. The zone of inhibition was compared with standard disc. The antimicrobial activity was recorded by measuring the width of the clear inhibition zone around the disc using zone reader $(\mathrm{mm})[23,24]$. The biological screening iseffective and the results show a significant antibacterial activity for the grown LYPB crystals over both Gram positive (Lactobacillus, Bacillus subtilis and Staphylococcus aureus) and Gram negative (Salmonella typhi and Escherichia coli) bacteria by agar diffusion method by measuring the inhibition zone width as shown in Fig 8.The inhibition zone diameters in the agar well diffusion assays for LYPB were given in Table 2 enabling a comparison[25,26].

Table 2. Zone of inhibition against select bacteria ( $\mathrm{mm})$

\begin{tabular}{|c|c|c|c|c|c|}
\hline \multirow{2}{*}{ Sample } & \multicolumn{5}{|c|}{ Zone of Inhibition (mm) } \\
\cline { 2 - 6 } & S.aureus & E.coli & B.subtilus & S.typhi & L.bacillus \\
\hline LYPB & $13 \pm 0.5$ & $12 \pm 0.2$ & $9 \pm 0.3$ & $10 \pm 0.1$ & $12 \pm 0.3$ \\
\hline LYSINE & $11 \pm 0.4$ & $16 \pm 0.3$ & $9 \pm 0.2$ & $12 \pm 0.3$ & $14 \pm 0.4$ \\
\hline
\end{tabular}

As compared to L-Lysine, the grown LYPB crystals show a moderate activity against the selected strains of microorganisms[27]. The sensitivity for five bacterial species follows the order as Staphylococcus aureus>Lactobacillus >Escherichia coli > Salmonella typhi >Bacillus subtitlis. Hence the grown LYPB crystals may be considered for pharmacological applications.

\section{Conclusion}

Single crystals of LYPB were developed using slow evaporation technique. Crystalline nature of developed compounds was verified with PXRD analysis and reflections were duly indexed. The lattice parameters were found by single crystal XRD. The grown single crystal LYPB had cubiccrystal system. The alteration inlattice parameters values proves the incorporation of L-lycine with KBr. For LYPB crystals the lower cut off wave length is around $246 \mathrm{~nm}$, transparency range is $80 \%$ and band gap energy is around 5.04 
Growth, characterization, and antibacterial studies of L-Lysine single crystals added with ..

$\mathrm{eV}$. Mechanical part of the analysis says that the grown crystals are soft materials. Antibacterial studies showed that the prepared crystals have moderate antibacterial activity against the select bacterial strains. From the above results, it is concluded that the grown crystals are suitable for biomedical applications. The grown crystals can also be used in the field of ferroelectric, optoelectronic and photonics.

\section{Acknowledgment}

The authors are thankful to Indian Institute of Science, Bangalore; Bharathiar University, PSG College of Arts and Science, Coimbatore; and St.Joseph College, Trichy; Nandha college of pharmacy,Perundurai, for extending their facilities during the course of this study.

\section{References}

[1]. K.Mohan, Priyadarshini,A.Chandramohan,G.AnandhaBabu,P.R. amasamy,Synthesis, crystal growth, spectral, optical, thermal and dielectricstudies of dichloro(4-hydroxy-1-proline)cadmium(II) single crystals Optik 125, 2014, 1390-1395.

[2]. B. Narayana Moolya, S.M. Darmaprakash, Nonlinear optical diglycine hydrochloride: synthesis, crystal growth and structural characteristics, J. Cryst. Growth, 293, 2006, 86-92.

[3]. M. Kumar, S. Tamilselvan, M. Vimalan, P. Saravanana, S. Aruna, R. Kanagadurai Dielectric and photoconductivity properties of semiorganic nonlinear optical LMHCl crystals , Archives of Applied Science Research, , 5 (5), 2013,220-227.

[4]. V Vasudevan, R Ramesh Babu, A Reicher Nelcy, G Bhagavannarayana And K Ramamurthi Synthesis, Growth, Optical, Mechanical And Electrical PropertiesOf L-Lysine L-Lysinium Dichloride Nitrate (L-Lldn) Single Crystal, Bull. Mater. Sci.,. 34,. (3) 2011, 469-475.

[5]. Sd. Zulifiqar Ali Ahamed, G.R. Dillip, P. Raghavaiah, K. Mallikarjuna,B. Deva Prasad Raju Spectroscopic and thermal studies of c-glycine crystal grown from potassium bromide for optoelectronic applications, Arabian Journal of Chemistry ,6, 2013 , 429-433.

[6]. A. Silambarasan, P. Rajesh, P. Ramasamy Study on structural, morphological, optical and thermal properties of guanidine carbonate doped nickel sulfate hexahydrate crystal, Spectrochimica Acta Part A： Molecular and Biomolecular Spectroscopy 134, 2015 ,345349 .

[7]. Dr. J. Thomas Joseph Prakash M. Lawrence Growth and Characterization of Pure and L-lysine Doped Zinc (TRIS) Thiourea Sulphate Crystals, International Journal of Computer Applications, 8(.3), 2010, 0975 - 8887.

[8]. Suresh Sagadevan, Priya Murugasen2 Optical And Dielectric Studies On Semiorganic Nonlinear Optical Crystal By Solution Growth Technique, International Journal of Recent advances in Physics (IJRAP), ,3(1), 2014.,

[9]. P.S. Kannan, A. Ramadoss, G. Ganesh, and A. SubbiahPandi, Synthesis, Growth and Characterization of a novel Organic crystal Glycine Potassium Iodide (GPI), Archives of Physics Research, 2 (4), 2011, 137-142.

[10]. Kanagasabapathy, R. Rajasekaran, Growth aspects, spectral, thermal and hardness studies of rare earthcerium(III)nitrate doped zinc(tris) thiourea sulphate single crystals, Optik,124, 2013 4240- 4245 .

[11]. C.K. Lakshmana Perumal a, A. Arulchakkaravarthi a, N.P. Rajesh a,P. Santhanaraghavan b, P. Ramasamy, Microhardness and slip systems of solution grown MHB crystals, Materials Letters, 56, 2002) 578-586.

[12]. N. Ravishankar, R. Chandramani,and A.P. Gnanaprakash Optical And Mechanical Characterization Of Solution Grown Semi Organic Nlo Crystals, Rasayan J. Chemistry Coden: Rjcabp, 4,(1) 2011, 86-90.

[13]. M. Bekte,s, O. Uzun, S. Akt"urk, A. E. Ekinci, and N. U,car Vickers Microhardness Studies Of Fe-Mn Binary Alloys, Chinese Journal Of Physics. 42,( 6), 2004.

[14]. R.Jothi Mani and P. Selvarajan Nucleation Kinetics, Growth, Nlo Studies, Hardness Parameters And Etching Analysis Of Phosphoric Acid Added L-Alanine Single Crystals (Pla), International Journal of ChemTech Research CODEN (USA): IJCRGG,6, (11), 2014, 4702-4708.

[15]. M. Iyanar, J. Thomas Joseph Prakash, C. Muthamizhchelvan and S. Ponnusamy Synthesis, Growth, and Characterization Studies of a Semiorganic Nonlinear Optical Single Crystal of Gamma Glycine, Journal of Physical Sciences, 13, 2009, 235-244

[16]. Mary Linet, S. Jerome Das, Optical, mechanical and transport properties of unidirectional grown 1-tartaric acid bulk single crystal for non-linear optical application, Materials Chemistry and Physics, 126,2011 886-890.

[17]. G. Babu Rao, P. Rajesh , P. Ramasamy,A study on the growth, optical, thermal, mechanical, dielectric and piezoelectric properties of dye doped KAP single crystals, Materials Research Bulletin, 60, 2014, 709-713

[18]. V. Sivashankara, R. Siddheswaran, P. Murugakoothan, Synthesis, growth, structural, optical and thermal properties of a new semiorganic nonlinear optical guanidinium perchlorate single crystal, Materials Chemistry and Physics ,130, 2011 323- 326.

[19]. S. Suresh and D. Arivuoli Growth, Optical, Mechanical and Dielectric Properties of Glycine Zinc Chloride NLO Single Crystals ,Journal of Minerals \& Materials Characterization \& Engineering, 10,(12), 2011,1131-1139.

[20]. P. Koteeswari, S. Suresh, P. Mani, Crystal Growth, Optical and Dielectric Properties of L-Histidine Hydrochloride Monohydrate Nonlinear Optical Single crystals, Journal of Minerals and Materials Characterization and Engineering,11, 2012, 813-816.

[21]. G. Maruthi, and R. Chandramani, Synthesis Of Solution Grown Efficient Semi Organic Nlo Crystals For Short Wave Length Generation - L-Arginine, L- Histadine With Additives, Rasayan J.Chemistry, 4,( 2), 2011, 280-284.

[22]. Uma shrma, Rajneesh K, Agnihotri, Showkat Ahmad, Surabhi Mahajan and Rajendra Sharma. (Antibacterial activity of some medicinal plants of family Lamiaceae from Braj region), Glob J Med Plant Res, 1(1), 2013,72-6.

[23]. Bayer, AW, Kirby, WMM, Sherris, JC \& Turck, M 1966, 'Antibiotic susceptibility testing by a standardized single disc method', American Journal of Clinical Pathology,. 45,.493-496.

[24]. Adegoke, A, Adebayo-tayo, A \& Bukola, C 2009, 'Antibacterial activity and phytochemical analysis of leaf extracts of Lasienthera africanum', African Journal of Biotechnology, 8,(1).77-80.

[25]. Peter R. Rodrigues and Satish M. Bhalekar. (Synthesis and biological Activity of 4-(4-Bromophenyl)-2-[4-(arylhydrazono-3methyl-5-(5-bromopyridin-2-Yl) imino-4,5-dihydro pyrazol-1-yl]-1,3-thiazole derivatives). Int. J. Chem. Sci. 13(4), 2015, 1844-5.

[26]. K. N. Puri and G. V. Korpe. (Synthesis, characterization and antimicrobial study of some benzoylated glucopyranosyl disubstituted thiocarbamides), Int. J. Chem. Sci., 13(4),2015, $1787 .$.

[27]. Sunil S. Patila, Ganesh A. Thakurb And Vishwanath R. Patil*C Synthesis, Spectral And Biological Studies On Some Mixed Ligand Ni(Ii) Complexes, Acta Poloniae Pharmaceutica N Drug Research,. 66, (3), $2009,27$.

[28]. K.Mohan, Priyadarshini, A.Chandramohan,G.AnandhBabu, P.Ramasamy, Optik 125, 1390 (2014)

[29]. B. Narayana Moolya, S.M. Darmaprakash, J. Cryst. Growth 293,86 (2006). 
Growth, characterization, and antibacterial studies of L-Lysine single crystals added with ..

[30]. M. Kumar, S. Tamilselvan, M. Vimalan, P. Saravanan, S. Aruna, R. Kanagadurai, Archives of Applied Science Research 5(5), 220 (2013).

[31]. V. Vasudevan, R. Ramesh Babu, A. Reicher Nelcy, G. Bhagavannarayanaand K. Ramamurthi,Bull. Mater. Sci 34, 469 (2011).

[32]. Sd. Zulifiqar Ali Ahamed, G.R. Dilip, P. Raghavaiah, K. Mallikatjuna, B. Deva Prasad Raju, Arabian Journal of Chemistry 6, 429 (2013)

[33]. A. Silambarasan, P. Rajesh , P. Ramasamy, Spectrochimica Acta Part A: Molecular and Biomolecular Spectroscopy 134,345 (2015)

[34]. Dr. J. Thomas Joseph Prakash, M. Lawrence, International Journal of Computer Applications 8 (3), 0975 (2010).

[35]. Suresh Sagadevan1, Priya Murugasen, International Journal of Recent advances in Physics 3(1), (2014).

[36]. P.S. Kannana, A. Ramadossb, G. Ganesha, and A. SubbiahPandi, Archives of Physics Research 2 (4), 137(2011).

[37]. Kanagasabapathy. R, Rajasekaran. P, Optik 124, 4240 (2013).

[38]. C.K. Lakshmana Perumal, A. Arulchakkaravarthi, N.P. Rajesh, P. Santhanaraghavan, P. Ramasamy, Materials Letters 56,578 (2002).

[39]. N. Ravishankar, R. Chandramani, and A.P. Gnanaprakash, Rasayan J. Chemistry 4 (1), 86 (2011).

[40]. M. Bekte, O. Uzun, S. Akt“urk, A. E. Ekinci,and N. U, Car Chinese Journal of Physics 42,(6) (2004).

[41]. R.Jothi Mani, and P. Selvarajan,International Journal of ChemTech Research 6, 4702 (2014).

[42]. M. Iyanar, J. Thomas Joseph Prakash, C. Muthamizhchelvan, and S. Ponnusamy, Journal of Physical Sciences 13, 235 (2009).

[43]. Mary Linet, S. Jerome Das, Materials Chemistry and Physics 126, 886 (2011).

[44]. G. Babu Rao, P. Rajesh, P. Ramasamy, Materials Research Bulletin 60, 709 (2014).

[45]. V. Sivashankara, R. Siddheswaran, P. Murugakoothan, Materials Chemistry and Physics 130,323 (2011).

[46]. S. Suresh and D. Arivuoli, Journal of Minerals \& Materials Characterization \& Engineering, 10 (12),113 (2011).

[47]. P. Koteeswari1 , S. Suresh, P. Mani, Journal of Minerals and Materials Characterization and Engineering 11, 813 (2012).

[48]. G. Maruthi, and R. Chandramani, Rasayan J.Chemistry 4 (2), 280 (2011).

[49]. Uma shrma, Rajneesh K, Agnihotri, Showkat Ahmad, Surabhi Mahajan and Rajendra Sharma. Glob J Med Plant Res 1(1), 72 (2013).

[50]. Bayer, AW, Kirby, WMM, Sherris, JC \& Turck, M American Journal of Clinical Pathology45,493(1966).

[51]. Adegoke, A, Adebayo-tayo, A \& Bukola, ,African Journal of Biotechnology 18 (1), 77(2009).

[52]. Peter R. Rodrigues and Satish M. Bhalekar ,Int. J. Chem. Sci 13 (4), 1844 (2015).

[53]. K. N. Puri and G. V. Korpe, Int. J. Chem. Sci 13 (4), 1787 (2015).

[54]. Sunil S. Patila, Ganesh A. Thakurb And Vishwanath R. Patil, Acta Poloniae Pharmaceutica N Drug Research 66(3), 271 (2009). 